\title{
Coordinatewise decomposition of group-valued Borel functions
}

\author{
by
}

Benjamin D. Miller (Los Angeles, CA)

\begin{abstract}
Answering a question of Kłopotowski, Nadkarni, Sarbadhikari, and Srivastava, we characterize the Borel sets $S \subseteq X \times Y$ with the property that every Borel function $f: S \rightarrow \mathbb{C}$ is of the form $f(x, y)=u(x)+v(y)$, where $u: X \rightarrow \mathbb{C}$ and $v: Y \rightarrow \mathbb{C}$ are Borel.
\end{abstract}

Given a set $X$, let $X^{<\mathbb{N}}=\bigcup_{n \in \mathbb{N}} X^{n}$. The length of $s \in X^{<\mathbb{N}}$ is the unique natural number $|s|$ such that $s \in X^{|s|}$. The restriction of $\alpha \in X^{\mathbb{N}}$ to $n$ is the sequence $\alpha \mid n \in X^{n}$ whose $i$ th coordinate agrees with that of $\alpha$, for all $i<n$. We say that $s$ is an initial segment of $\alpha \in X^{\mathbb{N}}$, or $s \subseteq \alpha$, if there exists $n \in \mathbb{N}$ such that $s=\alpha \mid n$. The basic clopen set associated with $s \in X^{<\mathbb{N}}$ is given by $\mathcal{N}_{s}=\left\{\alpha \in X^{\mathbb{N}}: s \subseteq \alpha\right\}$. The diagonal on $X$ is defined by $\Delta(X)=\{(x, x)$ : $x \in X\}$. Given a set $S \subseteq X \times Y$, let $S^{-1}=\{(y, x) \in Y \times X:(x, y) \in S\}$.

A graph on $X$ is an irreflexive, symmetric set $\mathcal{G} \subseteq X \times X$. A $\mathcal{G}$-path from $x$ to $y$ is a sequence $\left\langle x_{i}\right\rangle_{i \leq n} \in X^{<\mathbb{N}}$ such that $x=x_{0}, y=x_{n}$, and $\forall i<n\left(\left(x_{i}, x_{i+1}\right) \in \mathcal{G}\right)$. Such a path is a $\mathcal{G}$-cycle if $n \geq 3, x=y$, and $\left\langle x_{i}\right\rangle_{i<n}$ is injective. We say that $\mathcal{G}$ is acyclic if there are no $\mathcal{G}$-cycles. The graph metric associated with such a graph is given by

$d_{\mathcal{G}}(x, y)= \begin{cases}n & \text { if there is an injective } \mathcal{G} \text {-path }\left\langle x_{i}\right\rangle_{i \leq n} \text { from } x \text { to } y, \\ \infty & \text { if there is no } \mathcal{G} \text {-path from } x \text { to } y .\end{cases}$

Suppose that $E$ is an equivalence relation on $X$. The $E$-class of $x \in X$ is given by $[x]_{E}=\{y \in X: x E y\}$. The E-saturation of a set $B \subseteq X$ is given by $[B]_{E}=\{x \in X: \exists y \in B(x E y)\}$. We say that $B$ is $E$-invariant if $B=[B]_{E}$, and we say that $B$ is an E-complete section if $X=[B]_{E}$. A reduction of an equivalence relation $E$ on $X$ to an equivalence relation $F$ on $Y$ is a function $\pi: X \rightarrow Y$ such that $\forall x_{1}, x_{2} \in X\left(x_{1} E x_{2} \Leftrightarrow \pi\left(x_{1}\right) F \pi\left(x_{2}\right)\right)$.

2000 Mathematics Subject Classification: Primary 03E15; Secondary 28A05.

Key words and phrases: coordinatewise decomposition.

The author was supported in part by NSF VIGRE Grant DMS-0502315. 
An embedding is an injective reduction. A separating family for $E$ is a family of sets $B_{0}, B_{1}, \ldots \subseteq X$ such that

$$
\forall x, y \in X\left(x E y \Leftrightarrow \forall n \in \mathbb{N}\left(x \in B_{n} \Leftrightarrow y \in B_{n}\right)\right) .
$$

We say that an equivalence relation $E$ on a Polish space is smooth if there is a Borel reduction of $E$ to $\Delta\left(2^{\mathbb{N}}\right)$, or equivalently, if $E$ admits a Borel separating family. A transversal of $E$ is a set $B \subseteq X$ such that $\forall x \in X\left(\left|B \cap[x]_{E}\right|=1\right)$.

Suppose that $S \subseteq X \times Y, \Gamma$ is a group, and $f: S \rightarrow \Gamma$. A coordinatewise decomposition of $f$ is a pair $(u, v)$, where $u: X \rightarrow \Gamma, v: Y \rightarrow \Gamma$, and

$$
\forall(x, y) \in S(f(x, y)=u(x) v(y))
$$

While our main goal here is to study coordinatewise decompositions in the descriptive set-theoretic context, we will first study the existence of coordinatewise decompositions without imposing any definability restrictions.

For the sake of notational convenience, we will assume that $X \cap Y=\emptyset$. The graph associated with $S$ is the graph on the set $Z_{S}=X \cup Y$ given by $\mathcal{G}_{S}=S \cup S^{-1}$. The following fact was proven essentially by Cowsik, Kłopotowski and Nadkarni [1]:

Proposition 1. Suppose that $X, Y$ are disjoint, $S \subseteq X \times Y$, and $\Gamma$ is a non-trivial group. Then the following are equivalent:

(1) Every function $f: S \rightarrow \Gamma$ admits a coordinatewise decomposition.

(2) $\mathcal{G}_{S}$ is acyclic.

Proof. To see $\neg(2) \Rightarrow \neg(1)$, suppose that there is a $\mathcal{G}_{S^{-}}$cycle of the form $\left\langle x_{0}, y_{0}, x_{1}, y_{1}, \ldots, x_{n+1}\right\rangle$. Fix $\gamma_{0} \in \Gamma \backslash\left\{1_{\Gamma}\right\}$ and define $f: S \rightarrow \Gamma$ by

$$
f(x, y)= \begin{cases}\gamma_{0} & \text { if }(x, y)=\left(x_{0}, y_{0}\right) \\ 1_{\Gamma} & \text { otherwise }\end{cases}
$$

If $(u, v)$ is a coordinatewise decomposition of $f$, then

$$
\begin{aligned}
\gamma_{0} & =f\left(x_{0}, y_{0}\right) f\left(x_{1}, y_{0}\right)^{-1} \cdots f\left(x_{n}, y_{n}\right) f\left(x_{n+1}, y_{n}\right)^{-1} \\
& =\left(u\left(x_{0}\right) v\left(y_{0}\right)\right)\left(u\left(x_{1}\right) v\left(y_{0}\right)\right)^{-1} \cdots\left(u\left(x_{n}\right) v\left(y_{n}\right)\right)\left(u\left(x_{n+1}\right) v\left(y_{n}\right)\right)^{-1} \\
& =u\left(x_{0}\right) u\left(x_{1}\right)^{-1} \cdots u\left(x_{n}\right) u\left(x_{n+1}\right)^{-1}=u\left(x_{0}\right) u\left(x_{n+1}\right)^{-1}=1_{\Gamma},
\end{aligned}
$$

which contradicts our choice of $\gamma_{0}$.

To see $(2) \Rightarrow(1)$, let $E_{S}$ be the equivalence relation whose classes are the connected components of $\mathcal{G}_{S}$, fix a transversal $B \subseteq Z_{S}$ of $E_{S}$, and define

$$
B_{n}=\left\{z \in Z_{S}: \exists w \in B\left(d_{\mathcal{G}_{S}}(w, z)=n\right)\right\} .
$$

For each $z \in B_{n+1}$, let $g_{n}(z)$ denote the unique $\mathcal{G}_{S^{-}}$-neighbor of $z$ in $B_{n}$, and define $u: X \rightarrow \Gamma, v: Y \rightarrow \Gamma$ recursively by setting $u(x)=v(y)=1_{\Gamma}$ for $x, y \in B_{0}$, and

$$
u(x)=f\left(x, g_{n}(x)\right) v\left(g_{n}(x)\right)^{-1}, \quad v(y)=u\left(g_{n}(y)\right)^{-1} f\left(g_{n}(y), y\right)
$$


for $x, y \in B_{n+1}$. To see that $(u, v)$ is a coordinatewise decomposition of $f$, suppose that $(x, y) \in S$, and fix $n \in \mathbb{N}$ such that $g_{n}(x)=y$ or $g_{n}(y)=x$. If $g_{n}(x)=y$, then $u(x)=f(x, y) v(y)^{-1}$, thus $f(x, y)=u(x) v(y)$. If $g_{n}(y)=x$, then $v(y)=u(x)^{-1} f(x, y)$, thus $f(x, y)=u(x) v(y)$.

As a corollary of the proof of Proposition 1, we obtain a sufficient condition for the existence of Borel coordinatewise decompositions:

Corollary 2. Suppose that $X, Y$ are disjoint Polish spaces, $S \subseteq X \times Y$ is Borel, $\Gamma$ is a standard Borel group, $\mathcal{G}_{S}$ is acyclic, and $E_{S}$ has a Borel transversal. Then every Borel function $f: S \rightarrow \Gamma$ admits a Borel coordinatewise decomposition.

Proof. It is sufficient to check that the functions $u$ and $v$ constructed in the proof of Proposition 1 are Borel. Letting $B_{n} \subseteq Z_{S}$ ands $g_{n}: Z_{S} \rightarrow Z_{S}$ be as above, it follows from the fact that $\mathcal{G}_{S}$ is acyclic that

$$
\begin{aligned}
z \in B_{n+1} & \Leftrightarrow z \notin \bigcup_{i \leq n} B_{i} \text { and } \exists w \in B_{n}\left((z, w) \in \mathcal{G}_{S}\right) \\
& \Leftrightarrow z \notin \bigcup_{i \leq n} B_{i} \text { and } \exists ! w \in B_{n}\left((z, w) \in \mathcal{G}_{S}\right),
\end{aligned}
$$

and results of Suslin and Luzin (see, for example, Theorems 14.11 and 18.11 of Kechris [5] or Theorem 4.4.3 and Corollary 4.12.2 of Srivastava [8]) then imply that each of these sets is Borel. As

$$
\operatorname{graph}\left(g_{n}\right)=\mathcal{G}_{S} \cap\left(B_{n+1} \times B_{n}\right),
$$

it follows that $g_{n}$ is Borel as well (see, for example, Theorem 14.12 of [5] or Theorem 4.5.2 of [8]), and this easily implies that $u$ and $v$ are Borel.

Our main theorem is that the sufficient condition given above is also necessary to guarantee the existence of Borel coordinatewise decompositions:

Theorem 3. Suppose that $X, Y$ are disjoint Polish spaces, $S \subseteq X \times Y$ is Borel, and $\Gamma$ is a non-trivial standard Borel group. Then the following are equivalent:

(1) Every Borel function $f: S \rightarrow \Gamma$ admits a Borel coordinatewise decomposition.

(2) $\mathcal{G}_{S}$ is acyclic and $E_{S}$ admits a Borel transversal.

Proof. As $(2) \Rightarrow(1)$ follows from Corollary 2, we need only show (1) $\Rightarrow(2)$. Towards this end, suppose that (1) holds. As the map $f$ described in the proof of $\neg(2) \Rightarrow \neg(1)$ of Proposition 1 is clearly Borel, it follows that $\mathcal{G}_{S}$ is acyclic, thus $E_{S}$ is Borel (by Theorems 14.11 and 18.11 of [5] or Theorem 4.4.3 and Corollary 4.12 .2 of [8]).

Fix a non-trivial countable subgroup $\Delta \leq \Gamma$, endow $\Delta$ with the discrete topology, and endow $\Delta^{\mathbb{N}}$ with the corresponding product topology. Define 
$E_{0}^{\Delta}$ on $\Delta^{\mathbb{N}}$ by

$$
\alpha E_{0}^{\Delta} \beta \Leftrightarrow \exists n \in \mathbb{N} \forall m>n(\alpha(m)=\beta(m)),
$$

and define $F_{0}^{\Delta} \subseteq E_{0}^{\Delta}$ on $\Delta^{\mathbb{N}}$ by

$$
\alpha F_{0}^{\Delta} \beta \Leftrightarrow \exists n \in \mathbb{N} \forall m>n(\alpha(0) \cdots \alpha(m)=\beta(0) \cdots \beta(m)) .
$$

Let $\Delta$ act freely on $\Delta^{\mathbb{N}}$ by left multiplication on the 0th coordinate, i.e.,

$$
\delta \cdot \alpha=\langle\delta \alpha(0), \alpha(1), \alpha(2), \ldots\rangle .
$$

LEMMA 4. The action of $\Delta$ on $\Delta^{\mathbb{N}}$ induces a free action of $\Delta$ on $\Delta^{\mathbb{N}} / F_{0}^{\Delta}$.

Proof. It is enough to observe that

$$
\forall \delta \in \Delta \forall \alpha, \beta \in \Delta^{\mathbb{N}}\left(\alpha F_{0}^{\Delta} \beta \Rightarrow \delta \cdot \alpha F_{0}^{\Delta} \delta \cdot \beta\right),
$$

which is a trivial consequence of the definition of $F_{0}^{\Delta}$.

Suppose now that $F \subseteq E$ are Borel equivalence relations on a Polish space $X$. We say that $E$ is relatively ergodic over $F$ if there is no $F$-invariant Borel set $B \subseteq X$ such that both $B$ and $X \backslash B$ are $E$-complete sections.

LEMMA 5. $E_{0}^{\Delta}$ is relatively ergodic over $F_{0}^{\Delta}$.

Proof. Suppose, towards a contradiction, that $B \subseteq \Delta^{\mathbb{N}}$ is an $F_{0}^{\Delta}$-invariant Borel set such that both $B$ and $\Delta^{\mathbb{N}} \backslash B$ are $E_{0}^{\Delta}$-complete sections. As $B$ is an $E_{0}^{\Delta}$-complete section, it follows that $B$ is non-meager, thus there exists $s \in \Delta^{<\mathbb{N}}$ such that $B$ is comeager in $\mathcal{N}_{s}$. Define $C \subseteq \Delta^{\mathbb{N}}$ by

$$
C=\Delta^{\mathbb{N}} \backslash\left[\mathcal{N}_{s} \backslash B\right]_{E_{0}^{\Delta}}
$$

and observe that $C$ is an $E_{0}^{\Delta}$-invariant comeager Borel set and $\mathcal{N}_{s} \cap C \subseteq$ $B \cap C$. It only remains to show that $C \subseteq B$, which implies that $\Delta^{\mathbb{N}} \backslash \bar{B}$ is meager, contradicting the fact that $\Delta^{\mathbb{N}} \backslash B$ is an $E_{0}^{\Delta}$-complete section. Towards this end, given $\alpha \in C$, set $n=|s|$ and define $\delta \in \Delta$ by

$$
\delta=(s(0) \cdots s(n-1))^{-1}(\alpha(0) \cdots \alpha(n)) .
$$

Then $\alpha F_{0}^{\Delta}\langle s(0), \ldots, s(n-1), \delta, \alpha(n+1), \alpha(n+2), \ldots\rangle$, thus $\alpha \in B$.

Recall that $E_{0}$ is the equivalence relation on $2^{\mathbb{N}}$ given by

$$
\alpha E_{0} \beta \Leftrightarrow \exists n \in \mathbb{N} \forall m>n(\alpha(m)=\beta(m)) \text {. }
$$

LEMma 6. There is a Borel embedding $\pi_{1}: \Delta^{\mathbb{N}} \rightarrow 2^{\mathbb{N}}$ of $E_{0}^{\Delta}$ into $E_{0}$.

Proof. Fix an enumeration $\left(k_{n}, \delta_{n}\right)$ of $\mathbb{N} \times \Delta$. Define $\pi_{1}: \Delta^{\mathbb{N}} \rightarrow 2^{\mathbb{N}}$ by

$$
\left[\pi_{1}(\alpha)\right](n)= \begin{cases}1 & \text { if } \alpha\left(k_{n}\right)=\delta_{n}, \\ 0 & \text { otherwise }\end{cases}
$$

It is straightforward to check that $\pi_{1}$ is the desired embedding.

Now suppose, towards a contradiction, that $E_{S}$ has no Borel transversal. LEMma 7. There is a Borel embedding $\pi_{2}: 2^{\mathbb{N}} \rightarrow Z_{S}$ of $E_{0}$ into $E_{S} \mid X$. 
Proof. Suppose, towards a contradiction, that there is no Borel embedding of $E_{0}$ into $E_{S} \mid X$. As $E_{S}$ is Borel, so too is $E_{S} \mid X$. It follows from Theorem 1.1 of Harrington-Kechris-Louveau [3] that $E_{S} \mid X$ is smooth. Fix a Borel separating family $B_{0}, B_{1}, \ldots$ for $E_{S} \mid X$, and observe that the sets

$$
A_{n}=B_{n} \cup\left\{y \in Y: \exists x \in B_{n}((x, y) \in S)\right\}
$$

form a $\boldsymbol{\Sigma}_{1}^{1}$ separating family for $E_{S} \mid\left(X \cup \operatorname{proj}_{Y}[S]\right)$, where $\operatorname{proj}_{Y}: X \times Y \rightarrow Y$ denotes the projection function. It easily follows that $E_{S}$ has a $\sigma\left(\Sigma_{1}^{1}\right)$ separating family, thus Theorem 1.1 of [3] implies that $E_{S}$ is smooth. As $\mathcal{G}_{S}$ is acyclic, it follows from Hjorth [4] (see also Miller [7]) that $E_{S}$ admits a Borel transversal, which contradicts our assumption that it does not.

For $x_{1} E_{S} x_{2}$, we say that $z$ is $\mathcal{G}_{S}$-between $x_{1}$ and $x_{2}$ if $z$ lies along the unique injective $\mathcal{G}_{S}$-path from $x_{1}$ to $x_{2}$. Define $B \subseteq Z_{S}$ by

$$
B=\left\{z \in Z_{S}: \exists x_{1}, x_{2} \in \operatorname{rng}\left(\pi_{2} \circ \pi_{1}\right)\left(z \text { is } \mathcal{G}_{S^{-}} \text {-between } x_{1} \text { and } x_{2}\right)\right\} .
$$

As $\mathcal{G}_{S}$ is acyclic and $\operatorname{rng}\left(\pi_{2} \circ \pi_{1}\right)$ intersects every $E_{S^{-}}$class in a countable set, it follows that $B$ is Borel. As $E_{S} \cap\left(B \times \operatorname{rng}\left(\pi_{2} \circ \pi_{1}\right)\right)$ has countable sections, the Luzin-Novikov uniformization theorem (see, for example, Theorem 18.10 of [5] or Theorem 5.8.11 of [8]) ensures that it has a Borel uniformization $\pi_{3}: B \rightarrow \operatorname{rng}\left(\pi_{2} \circ \pi_{1}\right)$. We can clearly assume that $\pi_{3} \mid \operatorname{rng}\left(\pi_{2} \circ \pi_{1}\right)=$ id. Define $\pi: B \rightarrow \Delta^{\mathbb{N}}$ by

$$
\pi=\left(\pi_{2} \circ \pi_{1}\right)^{-1} \circ \pi_{3},
$$

and finally, define $f: S \rightarrow \Delta$ by

$$
f(x, y)= \begin{cases}1_{\Gamma} & \text { if } x \notin B \text { or } y \notin B \\ \delta & \text { if } x, y \in B \text { and } \delta \cdot \pi(y) F_{0}^{\Delta} \pi(x) .\end{cases}
$$

Now suppose, towards a contradiction, that there is a Borel coordinatewise decomposition $(u, v)$ of $f$.

Lemma 8. Suppose that $x, x^{\prime} \in B \cap X$ and $x E_{S} x^{\prime}$. Then:

(1) $u(x) u\left(x^{\prime}\right)^{-1} \in \Delta$.

(2) $u(x) u\left(x^{\prime}\right)^{-1} \cdot \pi\left(x^{\prime}\right) F_{0}^{\Delta} \pi(x)$.

Proof. Let $\left\langle x_{0}, y_{0}, \ldots, x_{n}, y_{n}, x_{n+1}\right\rangle$ be the unique injective $\mathcal{G}_{S}$-path from $x$ to $x^{\prime}$. To see (1), observe that, for all $i \leq n$,

$$
u\left(x_{i}\right) u\left(x_{i+1}\right)^{-1}=\left(u\left(x_{i}\right) v\left(y_{i}\right)\right)\left(u\left(x_{i+1}\right) v\left(y_{i}\right)\right)^{-1}=f\left(x_{i}, y_{i}\right) f\left(x_{i+1}, y_{i}\right)^{-1},
$$

thus $u\left(x_{i}\right) u\left(x_{i+1}\right)^{-1} \in \Delta$. Noting that

$$
u\left(x_{0}\right) u\left(x_{n+1}\right)^{-1}=u\left(x_{0}\right) u\left(x_{1}\right)^{-1} u\left(x_{1}\right) u\left(x_{2}\right)^{-1} \cdots u\left(x_{n}\right) u\left(x_{n+1}\right)^{-1},
$$

it follows that $u(x) u\left(x^{\prime}\right)^{-1} \in \Delta$. 
To see (2), observe that for all $i \leq n$,

$$
\begin{aligned}
u\left(x_{i}\right) u\left(x_{i+1}\right)^{-1} \cdot\left[\pi\left(x_{i+1}\right)\right]_{F_{0}^{\Delta}} & =f\left(x_{i}, y_{i}\right) f\left(x_{i+1}, y_{i}\right)^{-1} \cdot\left[\pi\left(x_{i+1}\right)\right]_{F_{0}^{\Delta}} \\
& =f\left(x_{i}, y_{i}\right) \cdot\left[\pi\left(y_{i}\right)\right]_{F_{0}^{\Delta}}=\left[\pi\left(x_{i}\right)\right]_{F_{0}^{\Delta}} .
\end{aligned}
$$

Setting $C_{i}=\left[\pi\left(x_{i}\right)\right]_{F_{0}^{\Delta}}$, it follows that

$$
\begin{aligned}
u\left(x_{0}\right) u\left(x_{n+1}\right)^{-1} \cdot C_{n+1} & =u\left(x_{0}\right) u\left(x_{1}\right)^{-1} \cdots u\left(x_{n}\right) u\left(x_{n+1}\right)^{-1} \cdot C_{n+1} \\
& =u\left(x_{0}\right) u\left(x_{1}\right)^{-1} \cdots u\left(x_{n-1}\right) u\left(x_{n}\right)^{-1} \cdot C_{n} \\
& \vdots \\
& =C_{0},
\end{aligned}
$$

thus $u\left(x_{0}\right) u\left(x_{n+1}\right)^{-1} \cdot\left[\pi\left(x_{n+1}\right)\right]_{F_{0}^{\Delta}}=\left[\pi\left(x_{0}\right)\right]_{F_{0}^{\Delta}}$.

Define $w: \Delta^{\mathbb{N}} \rightarrow \Gamma$ by $w=u \circ \pi_{2} \circ \pi_{1}$, fix a countable Borel separating family $\Gamma_{0}, \Gamma_{1}, \ldots \subseteq \Gamma$ for $\Gamma$, and define $n: \Delta^{\mathbb{N}} \rightarrow \Gamma$ by

$$
n(\alpha)=\min \left\{n \in \mathbb{N}: \exists \delta_{1}, \delta_{2} \in \Delta\left(\delta_{1} w(\alpha) \in \Gamma_{n} \text { and } \delta_{2} w(\alpha) \notin \Gamma_{n}\right)\right\} .
$$

Lemma 8 ensures that if $\alpha E_{0}^{\Delta} \beta$, then $w(\alpha) w(\beta)^{-1} \in \Delta$, thus

$$
\Delta w(\alpha)=\Delta w(\alpha) w(\beta)^{-1} w(\beta)=\Delta w(\beta),
$$

and it follows that $n(\alpha)=n(\beta)$. As $\pi_{3} \mid \operatorname{rng}\left(\pi_{2} \circ \pi_{1}\right)=\mathrm{id}$, Lemma 8 also ensures that $w(\alpha) w(\beta)^{-1} \cdot \beta F_{0}^{\Delta} \alpha$. It follows that if $\alpha=\delta \cdot \beta$, then $w(\alpha) w(\beta)^{-1}$ $=\delta$, thus $w(\alpha)=\delta w(\beta)$. Defining $A \subseteq \Delta^{\mathbb{N}}$ by

$$
A=\left\{\alpha \in \Delta^{\mathbb{N}}: w(\alpha) \in \Gamma_{n(\alpha)}\right\},
$$

it follows that $A$ is an $F_{0}^{\Delta}$-invariant Borel set and both $A$ and $\Delta^{\mathbb{N}} \backslash A$ are $E_{0}^{\Delta}$-complete sections, which contradicts Lemma 5.

Kłopotowski, Nadkarni, Sarbadhikari and Srivastava [6] have studied coordinatewise decomposition using another equivalence relation $L$ which, modulo straightforward identifications, is the equivalence relation whose classes are the connected components of the dual graph $\breve{\mathcal{G}}_{S}$ on $S$, consisting of all pairs $\left(\left(x_{1}, y_{1}\right),\left(x_{2}, y_{2}\right)\right)$ of distinct elements of $S$ such that either $x_{1}=x_{2}$ or $y_{1}=y_{2}$. The equivalence classes of $L$ are the linked components of $S$, and the linked components of $S$ are said to be uniquely linked if $\mathcal{G}_{S}$ is acyclic.

ConjeCture 9 (Kłopotowski-Nadkarni-Sarbadhikari-Srivastava [6]). Suppose that $X, Y$ are disjoint Polish spaces and $S \subseteq X \times Y$ is Borel. Then the following are equivalent:

(1) Every Borel function $f: S \rightarrow \mathbb{C}$ has a Borel coordinatewise decomposition.

(2) The linked components of $S$ are uniquely linked and $L$ is smooth. 
In light of Theorem 3 and the above remarks, the following observation implies that Conjecture 9 is indeed correct:

Proposition 10. Suppose that $X, Y$ are disjoint Polish spaces, $S \subseteq$ $X \times Y$ is Borel, and $\mathcal{G}_{S}$ is acyclic. Then the following are equivalent:

(1) $E_{S}$ admits a Borel transversal.

(2) L is smooth.

Proof. To see $(1) \Rightarrow(2)$, suppose that $E_{S}$ admits a Borel transversal $B \subseteq Z_{S}$. Let $\pi_{1}: Z_{S} \rightarrow Z_{S}$ be the function which sends $z$ to the unique element of $B \cap[z]_{E_{S}}$, and let $\pi_{2}=\operatorname{proj}_{X} \mid S$. Then $\pi_{1}$ is a Borel reduction of $E_{S}$ to $\Delta\left(Z_{S}\right)$ and $\pi_{2}$ is a Borel reduction of $L$ to $E_{S}$, thus $\pi_{1} \circ \pi_{2}$ is a Borel reduction of $L$ to $\Delta\left(Z_{S}\right)$, so $L$ is smooth.

To see $(2) \Rightarrow(1)$, suppose that $L$ is smooth, and fix a Borel reduction $\pi_{1}: S \rightarrow 2^{\mathbb{N}}$ of $L$ to $\Delta\left(2^{\mathbb{N}}\right)$. Put $Z=\operatorname{proj}_{X}[S] \cup \operatorname{proj}_{Y}[S]$. By the Jankovvon Neumann uniformization theorem (see, for example, Theorem 18.1 of [5] or Theorem 5.5.2 of [8]), there is a $\sigma\left(\boldsymbol{\Sigma}_{1}^{1}\right)$-measurable reduction $\pi_{2}: Z \rightarrow S$ of $E_{S} \mid Z$ to $L$, thus $\pi_{1} \circ \pi_{2}$ is a $\sigma\left(\boldsymbol{\Sigma}_{1}^{1}\right)$-measurable reduction of $E_{S} \mid Z$ to $\Delta\left(2^{\mathbb{N}}\right)$. It easily follows that there is a $\sigma\left(\boldsymbol{\Sigma}_{1}^{1}\right)$-measurable reduction of $E_{S}$ to $\Delta\left(2^{\mathbb{N}}\right)$, thus Theorem 1.1 of [3] implies that $E_{S}$ is smooth. As $\mathcal{G}_{S}$ is acyclic, it follows from [4] (see also [7]) that $E_{S}$ admits a Borel transversal.

Acknowledgements. I would like to thank Mahendra Nadkarni, who first inspired me to explore the problem of coordinatewise decomposition. I would also like to thank Andrés Caicedo, Clinton Conley, and the anonymous referee, who made stylistic suggestions and brought to my attention typos in earlier drafts of this paper.

\section{References}

[1] R. C. Cowsik, A. Kłopotowski, and M. G. Nadkarni, When is $f(x, y)=u(x)+v(y)$ ?, Proc. Indian Acad. Sci. Math. Sci. 109 (1999), 57-64.

[2] R. Dougherty, S. Jackson, and A. Kechris, The structure of hyperfinite Borel equivalence relations, Trans. Amer. Math. Soc. 341 (1994), 193-225.

[3] L. Harrington, A. Kechris, and A. Louveau, A Glimm-Effros dichotomy for Borel equivalence relations, J. Amer. Math. Soc. 3 (1990), 903-928.

[4] G. Hjorth, A selection theorem for treeable sets, preprint, 2007.

[5] A. Kechris, Classical Descriptive Set Theory, Grad. Texts in Math. 156, Springer, New York, 1995.

[6] A. Kłopotowski, M. Nadkarni, H. Sarbadhikari, and S. Srivastava, Sets with doubleton sections, good sets and ergodic theory, Fund. Math. 173 (2002), 133-158.

[7] B. D. Miller, Definable transversals of analytic equivalence relations, preprint, 2007. 
[8] S. Srivastava, A Course on Borel Sets, Grad. Texts in Math. 180, Springer, New York, 1998.

Department of Mathematics

University of California

520 Portola Plaza

Los Angeles, CA 90095-1555, U.S.A.

E-mail: bdm@math.ucla.edu

Received 19 January 200\%;

in revised form 25 June 2007 